\title{
EXTREME VALUES FOR THE SIDON CONSTANT
}

\author{
DONALD I. CARTWRIGHT, ROBERT B. HOWLETT AND JOHN R. MCMULLEN
}

\begin{abstract}
Let $G$ be a compact group and let $\phi \neq P \subseteq \hat{G}$. We consider the inequalities $1<\kappa(P)<\left(\Sigma_{\sigma \in P} d_{\sigma}^{2}\right)^{1 / 2}$, where $\kappa(P)$ denotes the Sidon constant of $P$. The condition $\kappa(P)=1$ essentially characterizes an example of Figa-Talamanca and Rider. The condition $\kappa(P)=\left(\Sigma_{\sigma \in P} d_{\sigma}^{2}\right)^{1 / 2}$ for finite $P$ is equivalent to the existence of certain interesting functions on $G$. We show that $\kappa(\hat{G})=|G|^{1 / 2}$ for a very large class of finite groups $G$, and this implies the existence of " $G$-circulant" unitary matrices whose entries all have modulus $|G|^{-1 / 2}$.
\end{abstract}

Let $G$ be a compact group and let $P$ be any nonempty subset of $\hat{G}$, the dual hypergroup of $G$. We denote by $\mathcal{T}_{P}(G)$ the space of trigonometric polynomials $f$ on $G$ whose Fourier transforms $\hat{f}$ vanish off $P$. The point of departure of this article is the easily proved pair of inequalities

$$
1 \leqslant \kappa(P) \leqslant\left(\sum_{\sigma \in P} d_{\sigma}^{2}\right)^{1 / 2}
$$

(see Proposition 1), in which $\kappa(P)$ denotes the Sidon constant of $P$, defined by

$$
\kappa(P)=\sup \left\{\|\hat{f}\|_{1}=\sum_{\sigma \in P} d_{\sigma}\|\hat{f}(\sigma)\|_{\phi_{1}}: f \in \mathcal{T}_{P}(G),\|f\|_{\infty}<1\right\},
$$

and where, as usual, $d_{\sigma}$ denotes the degree of the representation $\sigma$ in $\hat{G}$, and \|\|$_{\phi_{1}}$ denotes the trace-class norm.

We investigate conditions under which $\kappa(P)$ takes one of the two bounding values in (1). For the case $\kappa(P)=1$ we give a complete answer. Indeed, we show that the example of Figà-Talamanca and Rider [1] of a Sidon set $P$ constructed using the projections on the various factors of a product of unitary groups gives essentially the only situation in which $\kappa(P)=1$ can occur.

We give in Proposition 3 various necessary and sufficient conditions for $\kappa(P)=$ $\left(\sum_{\sigma \in P} d_{\sigma}^{2}\right)^{1 / 2}$ to hold. In Theorem 2 and its corollary, we show that this is the case whenever $P=\hat{G}$ and $G$ belongs to a large class of finite groups. This special case permits construction of certain generalized circulant Hadamard matrices, or equivalently certain elements of the group algebra $\mathbf{C G}$. It also strongly generalizes the result of Graham [2], who established this when $G$ is a finite abelian group.

Finally, we show by example that there exist two finite groups $A$ and $B$, a hypergroup isomorphism $\phi: \hat{A} \rightarrow \hat{B}$, and a set $P \subseteq \hat{A}$ such that $\kappa(P) \neq \kappa(\phi(P))$. Thus the result of Vrem [7], that whether a subset $P$ of $\hat{G}$ is a central Sidon set

Received by the editors March 17, 1980.

1980 Mathematics Subject Classification. Primary 43A46, 20C05, 05B20.

Key words and phrases. Sidon constant, Sidon set, circulant Hadamard matrix.

(c) 1981 American Mathematical Society 0002-9939/81/0000-0156/\$02.75 
depends only on the hypergroup $\hat{G}$ and not on the compact group $G$ giving rise to it, is unlikely to hold sway for general Sidon sets.

Unexplained notation will be that of Hewitt and Ross [3].

1. The basic inequalities. Henceforth when $P \subseteq \hat{G}$ we use the notation $\|P\|=$ $\sum_{o \in P} d_{\sigma}^{2}$. Then using [3, (D.51) and (28.43)] and the Cauchy-Schwarz inequality it is easy to obtain

Proposition 1. Let $G$ be a compact group and let $P \subseteq \hat{G}$ be a finite nonempty set. Then for each $f \in \mathcal{T}_{P}(G)$ we have

$$
\|f\|_{\infty}<\|\hat{f}\|_{1}<\|P\|^{1 / 2}\|f\|_{\infty}
$$

and hence

$$
1 \leqslant \kappa(P)<\|P\|^{1 / 2} .
$$

2. Sets $P$ with $\kappa(P)=1$. Let $G=\Pi_{\alpha \in A} G_{\alpha}$ be a Cartesian product in which each factor $G_{\alpha}$ is a unitary group. Let $\mathrm{pr}_{\alpha}$ denote the projection on the factor $G_{\alpha}$, and for each $\alpha \in A$ let $\sigma_{\alpha} \in \hat{G}_{\alpha}$ be the self-representation. In [1], Figà-Talamanca and Rider showed that $P=\left\{\sigma_{\alpha} \circ \operatorname{pr}_{\alpha} \mid \alpha \in A\right\}$ is a Sidon set. In fact it is plain that $\kappa(P)=1$. The following theorem characterizes situations of this kind.

THEOREM 1. Let $G$ be a compact group, and let $P$ be any subset of $\hat{G}$. Let $\phi_{P}$ denote the homomorphism $\Pi_{\sigma \in P} \sigma$ of $G$ into $\mathscr{U}_{P}=\Pi_{\sigma \in P} \mathcal{U}\left(H_{\sigma}\right)$. Then $\kappa(P)=1$ holds if and only if every element of $\mathcal{U}_{P}$ can be written $\lambda \phi_{P}(x)$, with $\lambda \in T$ and $x \in G$. In particular, if $P=\{1\} \cup Q$ with $1 \notin Q$, then the condition becomes: $\phi_{Q}: G \rightarrow \mathcal{Q}_{Q}$ is a surjection.

The proof requires the following technical lemma.

LEMMA. Let $G$ be a compact group, let $P \subseteq \hat{G}$ be finite, and let $U_{\tau} \in \mathcal{Q}\left(H_{\tau}\right)$ be given for each $\tau \in P$. Then there exist $x_{1}, \ldots, x_{n} \in G$ and $\mu_{1}, \ldots, \mu_{n} \in \mathbf{C}$ such that $\Sigma\left|\mu_{k}\right|=1$ and such that $U_{\sigma}=\kappa(P) \sum_{n=1}^{n} \mu_{k} \sigma\left(x_{k}\right)$, for each $\sigma \in P$. Indeed $\kappa(P)$ is the smallest constant with this property.

The lemma follows from the bipolar theorem applied to the inclusion $A \subseteq$ $\kappa(P) B^{00}$, in which $B=\left\{\bigoplus_{\sigma \in P} \sigma(x) \mid x \in G\right\}$ and $A=\left\{\bigoplus_{\sigma \in P} U_{\sigma} \mid U_{\sigma} \in \mathcal{U}\left(H_{\sigma}\right)\right.$ for all $\sigma \in P\}$ are regarded as subsets of $\mathcal{L}\left(\bigoplus_{\sigma \in P} H_{\sigma}\right)$, and the absolute bipolars are taken with respect to the pairing $\langle T, S\rangle=\operatorname{trace}(T S)$. (Notice that the circled convex hull $\Gamma(B)$ of $B$ is closed, since $B$ is compact and $\mathcal{L}\left(\bigoplus_{\sigma \in P} H_{\sigma}\right)$ is finitedimensional.)

Now the assertion of the theorem for finite $P$ follows immediately from the lemma, since $\mathcal{U}(H)$ is the set of extreme points of the unit ball of $\mathcal{L}(H)$ (see [5, Theorem 1.6.4]). The case of infinite $P$ then follows by a routine compactness argument.

Remark. If $G=\mathrm{T}$, then Theorem 1 shows that a subset $P$ of $\mathrm{Z}$ whose Sidon constant is 1 has at most two elements, since $\phi_{P}(G)$ has dimension at most one as a submanifold of $\mathscr{U}_{P}$, while the latter has dimension card $P$. Similarly, if $G$ is a 
compact Lie group of dimension $n$ and if $P \subseteq \hat{G}$ with $\kappa(P)=1$, then $P$ may contain at most $n+1$ elements.

Before leaving this topic we record without proof a curious geometric interpretation of the Sidon constant afforded by the above lemma. We let $d($, ) denote the metric obtained from the operator norm on $\mathcal{L}\left(\bigoplus_{\sigma \in P} H_{\sigma}\right)$, and define $\rho(P)=$ $\sup \{d(U, \Gamma(B)) \mid U \in A\}$ where $B, \Gamma(B)$ and $A$ are defined as in the proof of the lemma. Thus for a single $\sigma \in P$ we have

$$
\rho(\sigma)=\sup \left\{d(U, \Gamma(\sigma(G))) \mid U \in \mathcal{Q}\left(H_{\sigma}\right)\right\} .
$$

Proposition 2. $\kappa(P)=(1-\rho(P))^{-1}$ for any finite $P \subseteq \hat{G}$.

3. Sets $P$ with $\kappa(P)=\|P\|^{1 / 2}$.

Proposition 3. Let $G$ be a compact group and let $P \subseteq \hat{G}$ be nonempty and finite. Then $\kappa(P)=\|P\|^{1 / 2}$ holds if and only if there exists a complex-valued function $f$ on $G$ satisfying one of the following equivalent conditions:

(a) $f \in \mathcal{T}_{P}(G),\|\hat{f}\|_{1}=\|P\|^{1 / 2}$ and $\|f\|_{\infty}<1$.

(b) $|f| \equiv 1$, and $\|P\|^{1 / 2} \hat{f}(\sigma)$ vanishes for $\sigma \notin P$ and is unitary for $\sigma \in P$.

(c) $|f| \equiv 1$, and $f * f^{*}=\|P\|^{-1} \sum_{\sigma \in P} d_{\sigma} \chi_{\sigma}$.

Proof. Since $\mathcal{T}_{P}(G)$ is finite-dimensional, the mapping $f \mapsto\|\hat{f}\|_{1}$ attains its maximum on $\left\{f \in \mathscr{T}_{P}(G):\|f\|_{\infty} \leqslant 1\right\}$. Thus $\kappa(P)=\|P\|^{1 / 2}$ if and only if a function $f$ exists satisfying (a). When proving Proposition 1 one sees that for equality to hold in the inequality $\|\hat{f}\|_{1}<\|P\|^{1 / 2}\|f\|_{\infty}$ it is necessary and sufficient that $|f|$ be constant and that for some $c>0$ independent of $\sigma, c \hat{f}(\sigma)$ is unitary for all $\sigma \in P$. This shows the equivalence of (a) and (b). The equivalence of (b) and (c) is easily seen by taking Fourier transforms.

It will be convenient to write $\delta_{P}$ for the function $\|P\|^{-1} \Sigma_{\sigma \in P} d_{\sigma} \chi_{\sigma}$ appearing in (c). In a special situation, $\delta_{P}$ assumes a simple form:

Proposition 4. Let $N$ be an open normal subgroup of the compact group G. Let $\rho \in \hat{N}$ be $G$-invariant (i.e. the representation $\rho^{x}: n \mapsto \rho\left(x^{-1} n x\right)$ is equivalent to $\rho$ for each $x \in G)$. Let $P=\rho^{G}$, where we define $\rho^{G}=\left\{\sigma \in \hat{G}\right.$ : $\rho$ is a component of $\left.\left.\sigma\right|_{N}\right\}$. Then $P$ is finite, $\|P\|=[G: N] d_{\rho}^{2}$, and

$$
\delta_{P}(x)= \begin{cases}0 & \text { if } x \notin N, \\ \frac{\chi_{\rho}(x)}{d_{\rho}} & \text { if } x \in N .\end{cases}
$$

Proof. Put

$$
g(x)= \begin{cases}0 & \text { if } x \notin N, \\ \chi_{\rho}(x) & \text { if } x \in N .\end{cases}
$$

If $\sigma \in P$ we see that $\hat{g}(\sigma)=[G: N]^{-1} \int_{N} \chi_{\rho}(n) \sigma\left(n^{-1}\right) d n$ so that by Clifford's theorem $\hat{g}(\sigma)=[G: N]^{-1} d_{\rho}^{-1} I_{H_{\sigma}}$. If $\sigma \notin P$ then $\rho$ is not a component of $\left.\sigma\right|_{N}$ and therefore $\hat{g}(\sigma)=0$. From this we see that the equation $d_{\rho}[G: N] g=\Sigma_{\sigma \in P} d_{\sigma} \chi_{\sigma}$ holds, as both members are continuous functions and their Fourier transforms agree. Evaluation at the identity yields $[G: N] d_{\rho}^{2}=\|P\|$ and hence $d_{\rho} \delta_{P}=g$ follows. 
Our next theorem implies that $\kappa(P)=\|P\|^{1 / 2}$ holds for sets $P$ of the form $\rho^{G}$, at least when $G$ is a finite solvable group and $d_{\rho}=1$.

THEOREM 2. Let $N$ be a normal subgroup of a finite solvable group $G$, and let $\rho$ be a linear character of $N$ satisfying $\rho\left(x^{-1} n x\right)=\rho(n)$ for all $x \in G$ and $n \in N$. Then there exists a function $f: G \rightarrow \mathbf{C}$ satisfying

(i) $|f(x)|=1$ for all $x \in G$.

(ii) $f(x n)=f(x) \rho(n)$ for all $x \in G$ and $n \in N$.

(iii) $f * f^{*}=0$ off $N$.

Note. (i) and (ii) entail $\left.f * f^{*}\right|_{N}=\rho$.

Proof. The key idea is lifting $f$ from a subgroup $M_{1}$ to a subgroup $M_{3}$, where $M_{1} \triangleleft M_{2} \triangleleft M_{3}$ and $\left[M_{2}: M_{1}\right]=\left[M_{3}: M_{2}\right]=p$ a prime. Straightforward lifting of $f$ from $M_{1}$ to $M_{3}$ fails, in general.

Assuming the theorem is false, let $G$ be a counterexample of minimal order. Choose $N \triangleleft G$ and a $G$-invariant linear character $\rho$ of $N$ for which no function $f$ exists with the desired properties. We obtain a contradiction by means of several steps.

1. $\rho$ is faithful. Since $\rho$ is $G$-invariant, $H=\operatorname{ker} \rho$ is a normal subgroup of $G$. Let $\tilde{\rho}$ be the character of $N / H$ induced by $\rho$. If $H \neq\{1\}$, then by minimality of $G$ there exists $\tilde{f}: G / H \rightarrow C$ satisfying (i), (ii) and (iii) with $G$ replaced by $G / H$ and $N$ by $N / H$ and $\rho$ by $\tilde{\rho}$. Define $f: G \rightarrow \mathbf{C}$ by $f(x)=\tilde{f}(x H)$ and it is easy to see that $f$ has the desired properties. This contradiction shows that $H=\{1\}$.

2. $N$ is contained in the centre of $G$. This follows from step 1 since $\rho\left(x^{-1} n x\right)=$ $\rho(n)$ for all $x \in G$ and $n \in N$.

3. There do not exist proper subgroups $G_{1} / N, \ldots, G_{r} / N$ of $G / N$ such that each $\xi \in G / N$ is uniquely expressible as a product $\xi_{1} \xi_{2} \cdots \xi_{r}$ with $\xi_{j} \in G_{j} / N$ for each $j$. Suppose $G / N$ has such subgroups. Since $\left|G_{j}\right|<|G|$ for each $j$, there exist functions $f_{j}: G_{j} \rightarrow \mathbf{C}$ satisfying (i), (ii) and (iii) (with $G$ replaced by $G_{j}$ ). Our hypotheses and step 2 show that each $x \in G$ may be expressed in the form $x_{1} x_{2} \cdots x_{r}$ (with $x_{j} \in G_{j}$ for all $j$ ) in exactly $|N|^{r-1}$ ways. Property (ii) of the functions $f_{j}$ shows that a function $f: G \rightarrow \mathbf{C}$ is well defined if we set $f(x)=f_{1}\left(x_{1}\right) f_{2}\left(x_{2}\right) \cdots f_{r}\left(x_{r}\right)$ for any such decomposition of $x$. It is clear that $f$ satisfies properties (i) and (ii). To see that (iii) holds, notice that $f=(|G| /|N|)^{r-1} \tilde{f}_{1} * \cdots * \tilde{f}_{r}$, where

$$
\tilde{f}_{j}(x)= \begin{cases}0 & \text { if } x \in G \backslash G_{j}, \\ f_{j}(x) & \text { if } x \in G_{j} .\end{cases}
$$

Notice also that $\tilde{f}_{j} * \tilde{f}_{j}^{*}=\left[G: G_{j}\right]^{-1} \delta$, that $\delta * \delta=[G: N]^{-1} \delta$, where

$$
\delta(x)= \begin{cases}0 & \text { if } x \in G \backslash N, \\ \rho(x) & \text { if } x \in N,\end{cases}
$$

and that $\delta$ is central in $\mathscr{T}(G)\left(\delta=\delta_{P}\right.$ in the notation of Proposition 4). This completes step 3 .

4. $G / N$ is a p-group for some prime $p$. If $G / N$ is not a $p$-group, then being solvable it has nontrivial proper Hall subgroups $G_{1} / N$ and $G_{2} / N$ corresponding to 
complementary sets of prime divisors of $|G / N|$. Since $G / N=G_{1} / N \cdot G_{2} / N$ and $G_{1} / N \cap G_{2} / N=\{1\}$, step 3 yields a contradiction.

5. If $|N|>1$ then $\rho$ does not extend to a character of $G$. Suppose that $|N|>1$ and that $\rho$ extends to a character $\tilde{\rho}$ of $G$. By minimality of $G$ there exists a function $\tilde{f}: G / N \rightarrow$ C satisfying (i), (ii) and (iii) (with $G$ replaced by $G / N, N$ by the trivial subgroup of $G / N$ and of course $\rho$ by the trivial representation). Define $f: G \rightarrow \mathbf{C}$ by $f(x)=\tilde{f}(x N) \tilde{\rho}(x)$ and clearly a contradiction is obtained.

6. $G$ is not abelian. If $G$ is abelian, then $N=\{1\}$ by step 5. But then the set $P$ of Proposition 4 is the whole dual group $\hat{G}$ and so $\kappa(P)=\|P\|^{1 / 2}\left(=|G|^{1 / 2}\right)($ see Graham [2]). Hence a function $f$ exists satisfying (i), (ii) and (iii).

7. For the $p$-group $G / N$ we have $p=2$. By steps 2 and 6 we have $N \varsubsetneqq G$, and so there is a subgroup $M$ of $G$ with $N \subset M, M / N \subseteq Z(G / N)$ and $[M: N]=p$. Then $M$ is abelian and normal. Let $\Lambda=\left\{\lambda \in \hat{M}:\left.\lambda\right|_{N}=\rho\right\}$, and fix any $\lambda_{0} \in \Lambda$. Then $\Lambda=\left\{\lambda_{0} \nu: \nu \in N^{0}\right\}$, where $N^{0}$ denotes the subgroup of $\hat{M}$ whose elements are trivial on $N$. Now $G$ acts on $\Lambda$ and fixes $N^{0}$ elementwise. Let $H_{0}$ be the stabilizer of $\lambda_{0}$. Since $|\Lambda|=p$ we must have $H_{0}=G$ or $\left[G: H_{0}\right]=p$. Since $M$ is abelian, we have $M \subseteq H_{0}$ and $M \neq G$. Hence there is a subgroup $H$ of $G$ with $[G: H]=p$ and $M \subseteq H \subseteq H_{0}$. Since $|H|<|G|$ there is for each $\lambda \in \Lambda$ a function $f_{\lambda}: H \rightarrow C$ satisfying (i), (ii) and (iii) with $G$ replaced by $H, N$ replaced by $M$, and $\rho$ replaced by $\lambda$. Choose coset representatives $\left\{x_{\lambda}: \lambda \in \Lambda\right\}$ for $H$ in $G$ (note that $|\Lambda|=[G: H]=p$ ) and define $f: G \rightarrow C$ by $f\left(x_{\lambda} h\right)=f_{\lambda}(h)$ for $\lambda \in \Lambda$ and $h \in H$. Clearly $f$ satisfies (i) and (ii), and one calculates that

$$
\left(f * f^{*}\right)(x)= \begin{cases}0 & \text { if } x \notin M, \\ \frac{1}{p} \sum_{\lambda \in \Lambda} \lambda\left(x_{\lambda}^{-1} x x_{\lambda}\right) & \text { if } x \in M .\end{cases}
$$

(Note that $\lambda^{H} \cap \lambda^{\prime H}=\varnothing$ when $\lambda, \lambda^{\prime} \in \Lambda$ are distinct, since $\lambda$ is the only component of $\left.\sigma\right|_{M}$ if $\sigma \in \lambda^{H}$. Therefore $f_{\lambda} * f_{\lambda^{\prime}}^{*}=0$ if $\lambda \neq \lambda^{\prime}$, taking the convolution over $H$ here, since $f_{\lambda} \in \mathcal{T}_{Q}(H)$ with $Q=\lambda^{H}$.)

If the coset representatives $x_{\lambda}$ are chosen so that the characters $\left\{\lambda^{x_{\lambda}}: \lambda \in \Lambda\right\}$ are distinct, then the sum in (3) is simply $\Sigma_{\lambda \in \Lambda} \lambda(x)$ and so $f$ satisfies (iii). Such a choice of $x_{\lambda}$ 's is achieved as follows: Suppose first that $H_{0} \neq G$. Pick $x \in G \backslash H_{0}$. Thus $\lambda_{0}^{x}=\lambda_{0} \nu$ for some nontrivial $\nu \in N^{0}$. The powers $\nu^{k}$ of $\nu$ for $k=0, \ldots, p-$ 1 are distinct, and so $\lambda_{0} \nu^{k}$ runs through all of $\Lambda$. For each $k$ we have $\left(\lambda_{0} \nu^{k}\right)^{x^{k}}=$ $\lambda_{0} \nu^{2 k}$, and if $p \neq 2$ the characters $\nu^{2 k}, k=0, \ldots, p-1$, are all distinct. Hence if we set $x_{\lambda}=x^{k}$ whenever $\lambda=\lambda_{0} \nu^{k}$, we have achieved our aim. In the case $H_{0}=G$, we have $\lambda^{x}=\lambda$ for all $x \in G$ and $\lambda \in \Lambda$, and any choice of $x_{\lambda}$ 's will do. Hence $p \neq 2$ leads to a contradiction.

8. $G / N$ is not a 2-group. The argument used in step 7 breaks down if the two extensions $\lambda_{1}$ and $\lambda_{2}$ of $\rho$ to $M$ are interchanged by some $x \in G$. Assume then that this is the case, so that $H=H_{0}$ in the above notation. If $H$ is the only maximal subgroup of $G$ containing $M$, then $G / M$ is cyclic, and since $M / N \subseteq Z(G / N)$, $G / N$ is abelian. But then $G / N$ is cyclic by step 3 and the structure theory of abelian groups. But this and $N \subseteq Z(G)$ shows that $G$ is abelian, contradicting step 6. So let $K$ be another maximal subgroup of $G$ containing $M$, and let $L=H \cap K$. 
Pick any $x \in K \backslash H$. Then $K=L \cup L x$, and $x$ interchanges $\lambda_{1}$ and $\lambda_{2}$. Let $g_{1}, g_{2}$ : $L \rightarrow C$ satisfy (i), (ii) and (iii) with $L$ replacing $G, M$ replacing $N$, and $\lambda_{1}$ (resp. $\lambda_{2}$ ) replacing $\rho$. Define $f_{1}, f_{2}: K \rightarrow \mathbf{C}$ by $f_{1}(l)=g_{1}(l)$ for $l \in L, f_{1}(l x)=g_{1}(l), f_{2}(l)=$ $g_{2}(l)$, and $f_{2}\left(l x^{-1}\right)=-g_{2}(l)$. Then $f_{1}$ and $f_{2}$ satisfy (i) and (iii) (but not (ii)) with $K$ replacing $G, M$ replacing $N$ and $\lambda_{1}$ (resp. $\lambda_{2}$ ) replacing $\rho$. Also $f_{1} * f_{2}^{*}=0$, taking the convolution over $K$. The calculations needed here are not difficult. For example, if $\xi \in L x$, write $\xi=l_{0} x$ with $l_{0} \in L$, and we have

$$
\begin{aligned}
& \frac{1}{|K|} \sum_{k \in K} f_{1}(k) \overline{f_{1}\left(\xi^{-1} k\right)} \\
& \quad=\frac{1}{|K|} \sum_{l \in L}\left\{f_{1}(l) \overline{f_{1}\left(x^{-1} l_{0}^{-1} l\right)}+f_{1}(l x) \overline{f_{1}\left(x^{-1} l_{0}^{-1} l x\right)}\right\} \\
& \quad=\frac{1}{|K|} \sum_{l \in L}\left\{g_{1}(l) \overline{g_{1}\left(x^{-1} l_{0}^{-1} l x^{-1}\right)}+g_{1}(l) \overline{\left.g_{1}\left(x^{-1} l_{0}^{-1} l x\right)\right\}}\right. \\
& \quad=\frac{1}{|M||K|} \sum_{l \in L, m \in M}\left\{g_{1}(l m) \overline{g_{1}\left(x^{-1} l_{0}^{-1} l m x^{-1}\right)}+g_{1}(\lim ) \overline{g_{1}\left(x^{-1} l_{0}^{-1} l m x\right)}\right\}=0,
\end{aligned}
$$

using the property (ii) for $g_{1}$ and the fact that $x$ interchanges $\lambda_{1}$ and $\lambda_{2}$.

Now pick any $y \in H \backslash K$. Then $G=K \cup y K$, and we may define $f: G \rightarrow \mathbf{C}$ by $f(k)=f_{1}(k)$ and $f(y k)=f_{2}(k)$ if $k \in K$. It is routine to check that $f$ satisfies properties (i), (ii) and (iii).

CoROllary. Let $G$ be a finite group having solvable subgroups $G_{1}, \ldots, G_{r}(r>1)$ so that each $x \in G$ is uniquely expressible as a product $x_{1} \cdots x_{r}$ with $x_{j} \in G_{j}$ for each $j$. Then $\kappa(\hat{G})=\|\hat{G}\|^{1 / 2}=|G|^{1 / 2}$.

Proof. When $G$ is solvable this follows from Theorem 2 above, taking $N$ to be the trivial subgroup. The general case follows from the solvable case as in step 3 of the proof.

REMARK. We conjecture that every finite group can be decomposed in the above manner. If this conjective is true, then $\kappa(\hat{G})=|G|^{1 / 2}$ and Theorem 2 are valid for every finite group $G$.

4. Circulant matrices. Let $G$ be a finite group. We will say that a matrix $\left(\xi_{i j}\right)_{i, j=1}^{n}$ is $G$-circulant if there is an enumeration $x_{1}=e, x_{2}, \ldots, x_{n}$ of $G$ and a function $h$ : $G \rightarrow \mathrm{C}$ such that $\xi_{i j}=h\left(x_{i}^{-1} x_{j}\right)(1<i, j<n)$. If $G=\left(a^{k}\right)_{k=0}^{n-1}$ is cyclic and if $x_{j}=a^{j-1}$ for $j=1,2, \ldots, n$ and if we put $m_{j}=\xi_{1 j}$ then such a matrix takes the form

$$
M=\left(\begin{array}{lllllll}
m_{1} & m_{2} & \cdot & \cdot & \cdot & \cdot & m_{n} \\
m_{n} & m_{1} & m_{2} & \cdot & \cdot & \cdot & m_{n-1} \\
\cdot & & & & & & \\
\vdots & & & & & & \\
m_{2} & m_{3} & \cdot & \cdot & \cdot & m_{n} & m_{1}
\end{array}\right)
$$

and is circulant in the usual sense. 
TheOREM 3. Let $G$ be any finite group of order $n$. Then there exists a unitary matrix which is $G$-circulant, and whose entries all have modulus $n^{-1 / 2}$, if and only if $\kappa(\hat{G})=n^{1 / 2}$.

Proof. Apply Propositions 3 and 4 with $N$ the trivial subgroup of $G$. If $f$ is a function satisfying (c) of Proposition 3, then the matrix $\left(\xi_{i j}\right)$ with $\xi_{i j}=n^{-1 / 2} f\left(x_{i}^{-1} x_{j}\right)$ has the desired properties.

The proof of Theorem 2 shows that the matrix in Theorem 3 can be chosen to have the form $A / \sqrt{n}$, where the entries of $A$ are roots of unity. For cyclic $G$, this matrix is real-valued if and only if $A$ is an $n \times n$ circulant Hadamard matrix. Such matrices $A$ are known not to exist for $5<n<12100$. For $A$ to have $\pm 1, \pm i$ as entries, $n$ is still very restricted (see, e.g. [6]).

5. A small example. Let $D$ and $Q$ denote respectively the dihedral and quaternion groups of order 8 , presented by $D=\left\langle a, b \mid a^{4}=1=b^{2}, b^{-1} a b=a^{-1}\right\rangle$ and $Q=$ $\left\langle a, b \mid a^{4}=1, b^{2}=a^{2}, b^{-1} a b=a^{-1}\right\rangle$. Then $\hat{D}$ and $\hat{Q}$ are isomorphic as hypergroups (see McMullen and Price [4]), each consisting of four linear representations, and a two-dimensional representation. Denote the two-dimensional representation of $D$ by $\sigma_{1}$ and that of $Q$ by $\sigma_{2}$. Then explicit calculations reveal that $\kappa\left(\left\{1, \sigma_{1}\right\}\right)=2$ but $\kappa\left(\left\{1, \sigma_{2}\right\}\right)=\sqrt{5}$. The set $P_{2}=\left\{1, \sigma_{2}\right\} \subseteq \hat{Q}$ therefore satisfies the equality $\kappa(P)=$ $\|P\|^{1 / 2}$, while $P_{1}=\left\{1, \sigma_{1}\right\} \subseteq \hat{D}$ does not. Thus the Sidon constant is not preserved by hypergroup isomorphisms. We also note that $P_{2}$ is not of the special form $\rho^{G}$ introduced in Proposition 4.

\section{REFERENCES}

1. A. Figà-Talamanca and D. Rider, $A$ theorem of Littlewood and lacunary series for compact groups, Pacific J. Math 16 (1966), 505-514. MR 34 \#6444.

2. C. C. Graham, The Sidon constant of a finite abelian group, Proc. Amer. Math. Soc. 68 (1978), 83-84. MR 56 \# 16262.

3. E. Hewitt and K. A. Ross, Abstract harmonic analysis, II, Springer-Verlag, Berlin and New York, 1970.

4. J. R. McMullen and J. F. Price, Reversible hypergroups, Rend. Sem. Mat. Fis. Milano 47 (1977), 67-85.

5. S. Sakai, $C^{*}$-algebras and $W^{*}$-algebras, Ergebnisse der Math. und ihrer Grenzgebiete, Band 60 , Springer-Verlag, Berlin and New York, 1971.

6. R. J. Turyn, Complex Hadamard matrices, Combinatorial Structures and their Applications (Proc. Calgary Internat. Conf., Calgary, Alberta, 1969), Gordon and Breach, New York, 1970, pp. $435-437$. MR 42 \# 5821.

7. R. C. Vrem, Lacunarity on compact hypergroups, Math. Z. 164 (1978), 93-104.

Department of MAThematics, University of Sydney, Sydney, N.S.W. 2006, Australia 